\title{
A KrF-LASER EXCITED BY A CAPACITIVELY COUPLED LONGITUDINAL DISCHARGE
}

\author{
T. GERBER, P.J.M. PETERS and H.M.J. BASTIAENS \\ Department of Applied Physics, Twente University of Technology, Enschede, The Netherlands
}

Received 25 January 1985

\begin{abstract}
The performance of a $\mathrm{KrF}$ excimer laser, excited by a discharge produced in a quartz tube between two metallic electrodes at its end and the inner tube wall serving as a dielectric electrode, is described. The dielectric electrode is capacitively coupled to a metallic electrode surrounding the quartz tube coaxially. Laser output energies up to $0.9 \mathrm{~mJ}$ in pulses having a duration of $6 \mathrm{~ns}$ FWHM could be obtained at a driving voltage of $100 \mathrm{kV}$.
\end{abstract}

In the last few years considerable efforts have been performed to develop excimer laser systems providing output energies of joules up to kilojoules. Comparatively less attention has been paid to miniature systems which would find their application in e.g. spectroscopy and testing purposes. Though transverse discharge systems can be scaled down to a small size [1], other excitation schemes as longitudinal $[2,3]$ or capacitively coupled longitudinal [4] and transverse [5] discharges may be appropriate for excimer lasers with output energies ranging from several $10 \mu \mathrm{J}$ to some $\mathrm{mJ}$. These alternative excitation techniques offer a similar power deposition capability compared to that of conventional transverse discharge devices. Their realization, however, involves much less technical sophistication such as accurate electrode shaping or auxiliary preionization.

Our report deals with the performance of a KrF-excimer laser excited in a longitudinal, capacitively coupled discharge device in which the corrosive laser gas is contained in a quartz tube. Discharges in typical $\mathrm{He} / \mathrm{Kr} / \mathrm{F}_{2}$ mixtures at a pressure ranging from 0.5 to about 3 bar yielded laser action in $\mathrm{KrF}$ at a wavelength of $248 \mathrm{~nm}$ with output energies up to $0.9 \mathrm{~mJ}$ in pulses having a duration of 6 ns FWHM. The output energy obtained with our set up is considerably higher than that achieved by Newman [4] in a similar experiment and comparable to that recently achieved in a capacitively coupled transverse discharge device made out of $\mathrm{BaTiO}_{3}$ [5].

Fig. 1 shows the outline of the laser device (a) and the equivalent electrical circuit (b). The laser gas is contained in a $25 \mathrm{~cm}$ long quartz tube with an inner diameter of $4 \mathrm{~mm}$ and a wall thickness of $1 \mathrm{~mm}$. Two

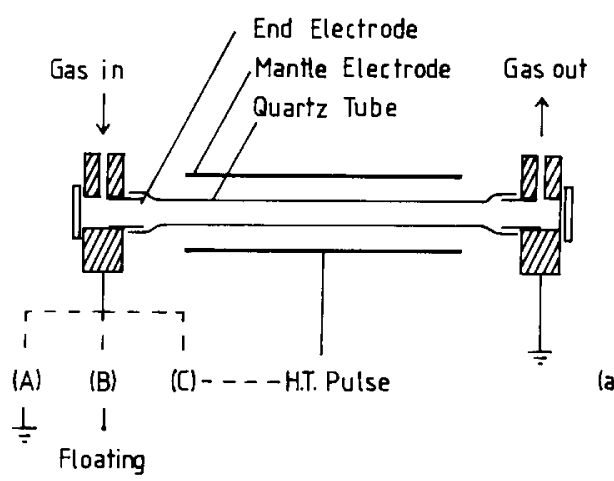

(a)

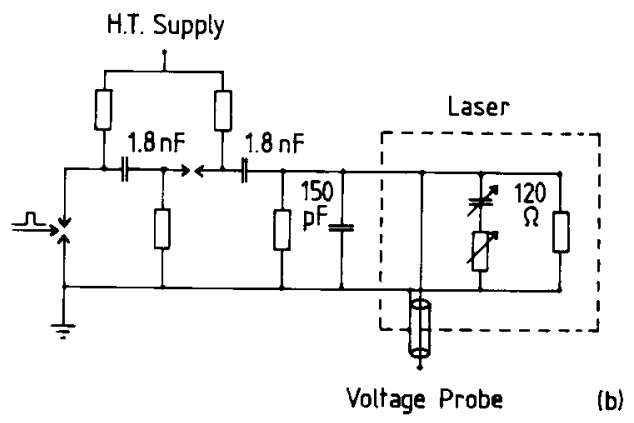

Fig. 1. Cross sectional illustration of the laser device (a), and equivalent electrical circuit (b) 
electrodes made out of stainless steel tubes with a 5 $\mathrm{mm}$ i.d. are inserted coaxially into the widened ends of the quartz tube. The electrodes are held in place by two flanges incorporating quartz windows and gas inlets. The windows can be adjusted to form a resonator. The flanges are sealed to the quartz tube with Viton O-rings. A third electrode is formed by a copper tube with $18 \mathrm{~mm}$ i.d. and a length of $180 \mathrm{~mm}$ which surrounds the quartz tube sharing the same axis. The gap between the copper- and quartz tube is filled with water isolating this mantle electrode to other parts of the discharge device.

The gas mixtures are premixed in a reservoir of a gas handling system and then fed into the laser device by means of copper or plastic tubings. For the measurements the laser gas fill was replaced by a fresh portion of the actual gas composite after a few shots.

A two stage Marx generator with a capacity of 1.8 $\mathrm{nF}$ per stage provided high voltage pulses up to $100 \mathrm{kV}$ with a risetime of about 20 ns. A plate capacitor of $150 \mathrm{pF}$ was connected in parallel with the laser electrodes serving as a peaking capacitor. A resistor of about $120 \Omega$ shortcircuiting the Marx generator truncates the voltage pulse to an overall duration of less than $300 \mathrm{~ns}$ in order to prevent dielectric breakdown of the quartz tube. Avoiding excessive gradients of the electric field along the laser tube by leaving a gap between the quartz surface and the mantle electrode the discharge device could withstand voltage pulses of 100 $\mathrm{kV}$ with superimposed peaks up to $300 \mathrm{kV}$.

In addition to the resonator formed by the two uncoated flat quartz windows a $100 \%$ dielectric reflector with a radius of curvature of $2 \mathrm{~m}$ was used. The distance between the two quartz flats is $27 \mathrm{~cm}$ and that between the quartz flat and the total reflector $10 \mathrm{~cm}$.

The laser output pulse energy was measured with a Gen Tech ED-100 pyroelectric energy meter. The pulse duration was monitored by a EG\&G FND 100 Q photodiode having a subnanosecond response. The voltage across the laser electrodes was detected with a $\mathrm{NaCl}$ voltage divider.

In a CC-discharge device the power coupled in to the discharge is limited by the capacity of the dielectric electrodes, i.e., the inner tube wall in respect to the outer metallic electrode, and the breakdown voltage of the gas in which a discharge is produced [6]. For a constant breakdown voltage the power deposited in the discharge is proportional to the voltage drop ac- cross the coupling capacity. The energy dissipated in the discharge during a complete discharge cycle increases in principle with the square of the applied voltage. Thus, for the dielectric electrode a material with a high dielectric constant and a high dielectric strength is desired. The dielectric constant of quartz is only 4 . But quartz offers the advantage of a high dielectric strength and a good chemical resistance to fluorine. Water having a dielectric constant of about 80 was chosen as an isolator in the gap between mantle electrode and laser tube in order to increase the coupling capacity of the dielectric electrode.

The measurements were started with the two electrodes inside the quartz tube grounded and charging the man tle electrode to negative potential (configuration (A) of fig. 1). In this configuration a discharge can take place between the end electrodes and the inner wall of the quartz tube serving as a capacitively ballasted dielectric electrode. The discharge is maintained until the potential between the end electrodes and the tube wall is too small to produce any further ionization in the contained gas, i.e., until the coaxial capacity formed by the conducting gas and the mantle electrode is charged to almost the full potential applied. When a voltage pulse arrives at the discharge device the gas between the end electrodes and the nearest quartz wall elements will break down. The corresponding gas volume is then in a conducting state and can be considered as an extension of the metallic end electrode. Therefore, a discharge can take place between this extended electrode and subsequent wall elements. Thus, the discharge starts at the end electrodes and expands into the quartz tube at a speed which essentially is given by the ionization rate achieved in the actual gas fill.

Fig. 2 shows the laser output energy at a wavelength of $248 \mathrm{~nm}$ obtained with three typical $\mathrm{He} / \mathrm{Kr} / \mathrm{F}_{2}$ mixtures as a function of total pressure. These measurements were performed with the Marx generator charged to $30 \mathrm{kV}$. Experiments with gas mixtures containing $0.25-1 \% \mathrm{~F}_{2}$ and $5-10 \% \mathrm{Kr}$ all produced similar results as that shown in fig. 2 yielding maximum output energies in the order of $400 \mu \mathrm{J}$. The optimum fill pressure decreases from 1.4 bar to 0.9 bar if the fluorine content is changed from $0.25 \%$ to $1 \%$. Thus, the best performance is found for partial fill pressures of about 6 mbar $F_{2}$ and about 50 mbar Kr. Slightly higher partial pressures are appropriate for higher excitation rates, 


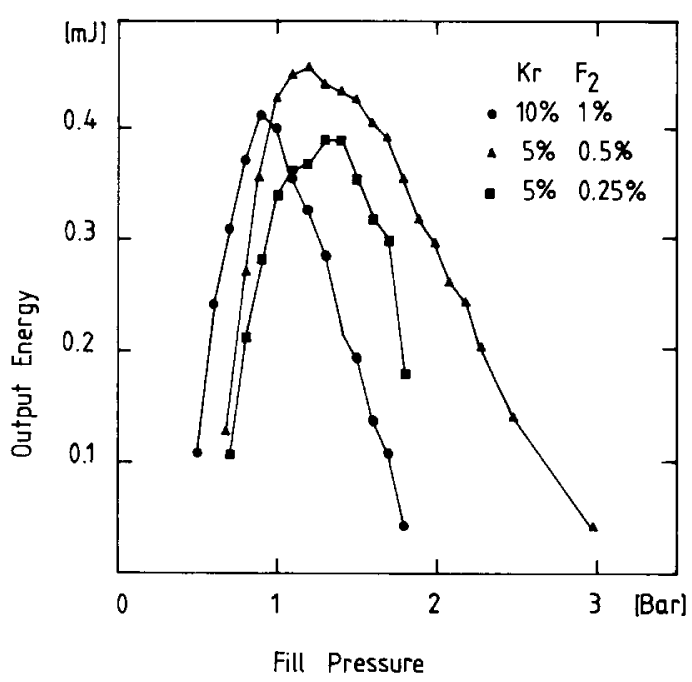

Fig. 2. Laser output energy obtained with three typical $\mathrm{He} / \mathrm{Kr} / \mathrm{F}_{2}$ mixtures as a function of total fill pressure.

i.e. for a higher initial Marx voltage. With all gas mixtures at all pressures the laser beam exhibits a ring pattern.

Fig. 3 shows the temporal behaviour of the voltage pulse applied to the laser and its correlation with the laser pulse having a duration of 6 ns FWHM. The peak observed at the leading edge of the voltage pulse, exceeding more than six times the Marx generator load voltage, is due to cumulating peaking effects at the

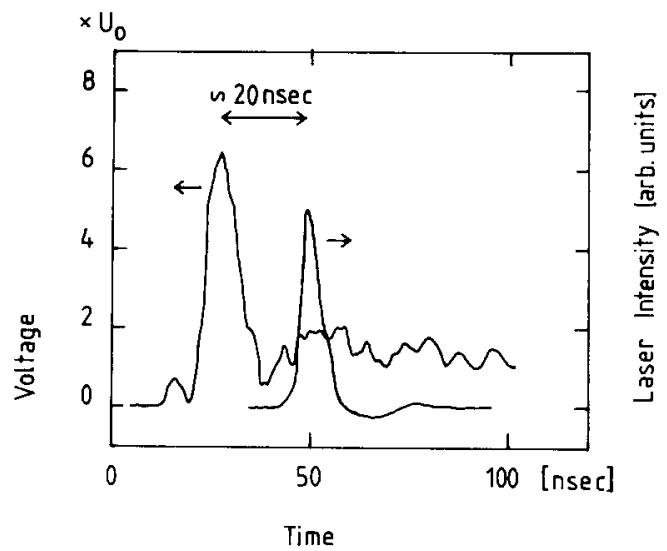

Fig. 3. Temporal behaviour of the laser pulse and of the voltage pulse arriving at the metallic electrodes of the discharge system. Unit of the voltage is the Marx load voltage $U_{0}$. peaking capacitor and at the capacity of the laser electrodes. The laser pulse is delayed by about $20 \mathrm{~ns}$ in respect to this voltage peak when the fill pressure is above $1 \mathrm{bar}$. The delay time increases up to $25 \mathrm{~ns}$ as the fill pressure is lowered to 0.6 bar. The lag between voltageand laser pulse does not correspond to the build-up time for laser action. The gain at the laser wavelength achieved with the described discharge device is very high as lasing could also be obtained with a resonator consisting of only the two window quartz flats. Thus, only very few roundtrips in the $1.2 \mathrm{~ns}$ long resonator are needed to produce laser output. We therefore have to conclude that the observed time delay is mainly due to the switching time of the discharge.

In the course of the measurements it was found that about $25 \%$ higher output energies can be achieved if the end electrode next to the outcoupling mirror has a floating potential (configuration (B) of fig. 1a). That is, a discharge takes place between only one end electrode and the dielectric wall electrode. At the floating electrode only a weak corona like discharge could be observed. The higher output obtained with this configuration can be due to the higher field strength along the plasma because of the higher discharge resistance and -inductivity in the presence of only one end electrode. Moreover the extraction efficiency may favorably be affected by a travelling wave excitation effect which possibly occurs when the discharge burns down the quartz tube in only one direction.

Fig. 4 summarizes the results with the discharge configuration described above. The gas mixture used contained $1 \% \mathrm{~F}_{2}$ and $10 \% \mathrm{Kr}$. Parameter is the initial Marx load voltage. As for these experiments plastic tubing had to be used between the gas inlet at the floating electrode and the gas handling system, the laser gas degrades more rapidly than in the initial set up. The maximum energy of $0.9 \mathrm{~mJ}$ achieved at a Marx generator load voltage of $50 \mathrm{kV}$ corresponds to a laser output power of $150 \mathrm{~kW}$. With the gas volume between the end electrodes being $2.5 \mathrm{~cm}^{3}$ the specific output energy would be $0.36 \mathrm{~J} / 1$. As laser action takes place only in a small region adjacent to the quartz tube wall the actual specific energy is much higher than this value.

Denoting the capacity of the pulser circuit with $C_{0}$ and its initial voltage with $U$ the maximum energy deposited in the laser gas is, neglecting losses, given by $E=\left(U^{2} / 2\right) C_{0} C /\left(C_{0}+C\right)$, 


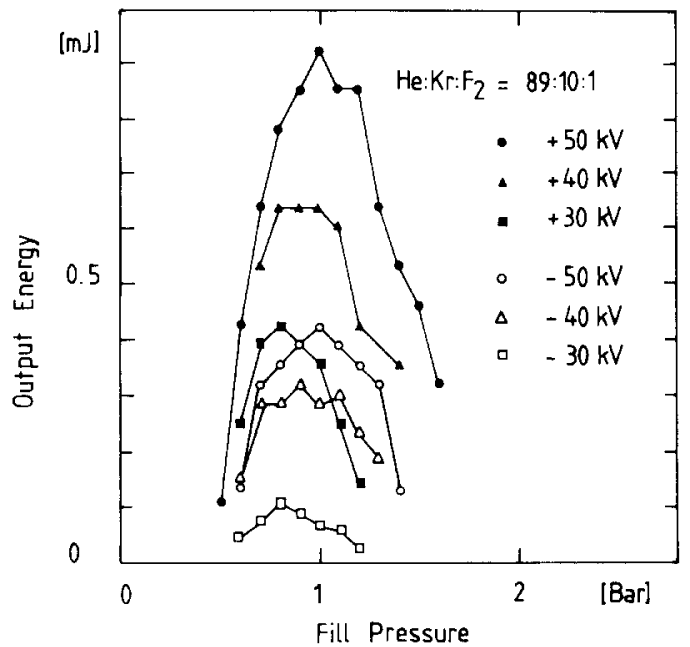

Fig. 4. Laser output energy obtained with a $\mathrm{He} / \mathrm{Kr} / \mathrm{F}_{2}$ mixture containing $10 \% \mathrm{Kr}$ and $1 \% \mathrm{~F}_{2}$ as a function of total pressure. Parameter is the Marx generator load voltage.

with $C$ being the laser capacity. In our set up $C$ is calculated to be $85 \mathrm{pF}$. $C_{0}$ corresponds in essence to the capacity of the switched Marx generator and has a value of $0.9 \mathrm{nF}$. Assuming an effective discharge voltage of twice the Marx generator load voltage, according to fig. 3 , the available excitation energy is for a charge voltage of $50 \mathrm{kV}$ about $390 \mathrm{~mJ}$. The poor efficiency of the laser device in the order of $0.25 \%$ indicates, that only part of this available energy is dissipated in the laser medium during the lasing cycle. Moreover the laser output energy does not increase linearly with the square of the Marx load voltage. Thus, saturation effects given either by kinetical limitations or by the transition to a less favorable discharge regime are present. In spite of that, still higher pulse energies could be obtained by a further increase of the applied voltage as the output increases more than linearly with the voltage in the investigated domain.

The laser output strongly depends on the polarity of the discharge electrodes. Using the dielectric electrode as anode less than half of the output energy could be achieved compared to the measurements with the quartz tube wall being the cathode. In both cases the laser beam exhibits a ring pattem. Thus, the volume in which the excited $\mathrm{KrF}$ complexes reach a density above laser threshold is restricted to a region close to either the anode or the cathode, respectively. The corresponding differences of the laser output may there- fore be attributed to changes of the $\mathrm{KrF}$ formation kinetics due to different discharge mechanisms near the cathode or the anode. Moreover the lasing volume and hence the extraction efficiency can be different in the two cases.

Combining the capacitively coupled discharge with a conventional longitudinal discharge by connecting one end electrode to the mantle electrode (configuration (C), fig. 1a) yielded no changes compared to the measurements performed with configuration (B). For fill pressures above 1 bar the temporal behaviour of the voltage pulse remains the same. Thus, in spite of the gas being ionized by the capacitively coupled discharge, probably no discharge strikes between the two end electrodes. At lower fill pressures a direct coupled Iongitudinal discharge takes place as can be seen by the more rapidly truncated voltage pulse as well as by visual inspection of the discharge itself which in this case is much brighter. But still, no effects such as a higher laser output energy, a longer pulse duration or a full beam pattern could be observed, indicating that this discharge mode is not suited to form and excite $\mathrm{KrF}$ molecules.

In conclusion we have shown that a capacitively coupled longitudinal discharge driven at a high voltage is suited to yield laser action in $\mathrm{KrF}$ at a wavelength of $248 \mathrm{~nm}$ with pulse energies up to $0.9 \mathrm{~mJ}$. The prominent features of the described laser system are its technical simplicity, the high gain and the high specific laser energy obtained with $\mathrm{KrF}$ as the lasing medium.

We would like to thank Prof. W.J. Witteman for his stimulating interest in our work. This experiment was partially supported by the Dutch Stichting voor Fundamenteel Onderzoek der Materie.

\section{References}

[1] R.C. Sze, J. Appl. Phys. 54 (1983) 1224.

[2] D. Cleschinsky, D. Damasch, H.J. Eichler and J. Hamisch, Optics Comm. 39 (1981) 79.

[3] Z. Zhou, Y. Zeng and M. Qiu, Appl. Phys. Lett. 43 (1983) 347.

[4] L.A. Newman, Appl. Phys. Lett. 33 (1978) 501.

[5] T. Gerber, H.M.J. Bastiaens and P.J.M. Peters, A miniature high power $\mathrm{KrF}$ laser excited with a capacitively coupled discharge, IEEE J. Quantum Electr. (1985) to be published.

[6] C.P. Christensen, Appl. Phys. Lett. 34 (1979) 211. 